XIV. Oeffentliches Sanitätswesen.

\section{Zur Verbreitung von Infectionskrankheiten durch den Genuss roher Milch.}

\section{Von}

Dr. A. Baginsky in Berlin.

Die No. 1309 (vom 30. Januar d. J.) des British medical Journal enthält einen interessanten Bericht von $\mathrm{A}$. Wynter Blyth über den Ausbruch einer Epidemie von Scharlach und Halsentzündung in Dorset Square, welche nach genauer Erforschung der Krankheitsquelle nur auf den Genuss inficirter Milch zurückgeführt werden konnte. - Die ersten etwas zahlreicher auftretenden Krankheitsfälle wurden am 14. December von Dr. Hickmann gemeldet. Schon am 9. December hatte Blyth einen Milchburschen wegen Scarlatina ins Krankenhaus geschickt. Er vermuthete eine Infection der Milch durch diesen Burschen, da die Erkrankungsfälle in Haushaltungen vorkamen, welche die Milch aus dem Verschleiss bezogen, in welchem der Milchbursche angestellt war. Die Vermuthung bestätigte sich nicht, vielmehr stellte sich heraus, dass der Bursche von der Milch selbst inficir worden war. Der Verschleiss bezog die Milch aus 2 Quellen. Die eine derselben war, wie Dr. P ower ermittelte, eine Farm (Hendon), in welcher seit dem 3. December mehrere Fälle von Scarlatina vorgekommen waren. Die Infectiosität der Milch dieser Farm stellte sich am deutlichsten dadurch heraus, dass in einigen armen Familien, welche trotz des Verbotes des weiteren Gebrauches der Milch von derselben genossen hatten, Scharlach auftrat. Eingehende Untersuchungen über die Beschaffenheit der Kühe, der Ställe und der Milch wurden auf Veranlassung von $\mathrm{Buchanan}$ und P ower durch Dr. Klein geführt und sollen noch mitgetheilt werden. Die Zahl der Erkrankungen betrug 60 . Sämmtliche Patienten hatten von der Milch der Hendomfarm genossen. Nur 3 Fälle von Scarlatina kamen in derselben Zeit in der Gegend vor, bei welchen der Genuss der inficirten Milch ausgeschlossen werden konnte. Die Erkrankungsfälle waren zum Theil leicht, nur. Anginen oder milde Scarlatina, zum Theil recht schwer. Ein Fall endete tödtlich. Die Incubationsdauer war sehr kurz, so erkrankte ein Kind 2 Tage nach dem Milchgenuss, ein junger Mann nach 3 Tagen.

Diejenigen, welche die Milch nur abgekocht genossen hatten, elkrankten nicht und auch die Meisten derjenigen, welche nur ein wenig Milch in Thee oder Kaffee genommen hatten, blieben frei. Die Hauptmasse der Erkrankten bilden Kinder, welche erbebliche Mengen lauwarmer oder ungekochter Milch und Erwachsene, welche robe Milch genossen liatten. Als besonders gefährlich zeigte sich der Milchrahm. - Verf. spricht die Vermuthung aus, dass die genau erforschte Epidemie möglicherweise Aufschlus über die Ursache der Scarlatina verschaffen wird.

So der Bericht. - In einem Briefe an den Herausgeber der Lancet beklagt zwar $\mathrm{J}$ ames $\mathrm{Cameron}$, der Officer of Health in Hendon, die etwas voreiligen Mittheilungen über die Epidemie, lässt indess erkennen, dass dieselben keineswegs alle, oder auch nur annähernd alle Thatsachen enthalten, welche sich betreffs der Beziehungen zwischen dem Milchgenuss un der Scarlatina in der betreffenden Epidemie häuften.

Wäre eine solche Mittheilung in der Literatur vereinzelt, so könnte dieselbe einigermaassen ungläubig aufgenommen werden. Indess liegen im Verlaufe der Jahre so reichhaltige Nachrichten über die Verbreitung epidemischer und infectiöser Krankheiten durch den Genuss roher Milch in der Literatur vor, dass es verlohnt, dem Gegenstande ernste Aufmerksamkeit zuzuwenden. Dies thut um so mehr Noth, als in der letzten Zeit in grossen Städten und insbesondere in Berlin die Neigung sich kund giebt, Kindern in den Schulen und auf öffentlichen Spielplätzen oder in Gärten rohe Milch als Getränk und Nahrungsmittel darzubieten und hierbei sogar die Idee vorwaltet, dass man den Kindern etwas ganz besonders Gesundheitsgemässes und Erspriessliches biete. In wohlgemeinter Absicht ist auch das Project aufgetaucht, die öffentlichen Milchhallen namentlich in den kinderreichen Vorstädten Berlins zu vermehren. - Der folgende Rückblick in die Literatur des Gegenstandes aus dem letzten Jahrzehnt hat nicht die Absicht, auf Vollständigkeit Anspruch zu machen, es sollen vielinehr nur die wichtigsten und am besten erwiesenen Thatsachen recapitulirt werden Vorzugsweise sind es drei Krankheitsformen, deren Verbreitung durch inficirte Milch in Frage kommen: Scharlach, Typhus und Tuberculose. Für die Frage der Infection von Kindern durch Milch von solchen Kühen, welche an Maul- und Klauenseuche litten, liegen bisher nur vereinzelte, aber immerhin ebenfalls bemerkenswerthe Mittheilungen vor; sehr schwankend und ernster Kritik nicht Stand haltend, sind die Mittheilungen über die Verbreitung der Diphtherie durch die Milch.

1) Scharlach

In meinem Berichte über die acuten Exantheme (Schmidt's Jahrbücher Bd. 175 Heft 2 p. 188) konnte ich auf Mittheilungen von. Ullersberger Bezug nehmen (Baier. ärztliches Intelligenzblatt 14. 1871), in welchen über Epidemien berichtet wird, welche nach Beobachtungen von Oswald Home, Bell und Taylor durch Milch entstanden ev. verbreitet worden waren. In dem Falle von Bell war die Krankheit von einer Meierei und deren Molkerei in die Universität St. Andrews hineingeschleppt worden 1) durch die Milch, 2) durch die Milchutensilien, 3) durch die Milchjungen, welche die Milch nach der Anstalt brachten. Von dieser Quelle gingen 26 Fälle aus, davon 2 tödtlich; für T aylor's Fälle stellte sich heraus, dass in 7 Häusern, wohin die Milch von einer Molkerei gebracht worden war, Scharlach zum Ausbruch kam und ein anderes Mal (1867) brach Scharlach in 6 Häusern aus, wohin die Milch von einem Milchmanne gebracht worden war, dessen Kinder an Scharlach darniederlagen. Es erkrankten nach einander Kinder und Erwachsene, welche die Milch genossen. - Auf dem medicinischen Congress in London berichtet Ernst Hart über 14 Scharlachepidemien, welche nachweislich durch die Milch verbreitet worden waren. Dieselbe umfassen 800 Scharlachfälle und Verf. glaubt, dass gelegentlich der Desquamation von Scarlatinekranken der Infectionskeim in die Milch gelange und weitergetragen werde. In den meisten dieser Epi demien liess sich auch nachweisen, dass Personen, die in der Milchwirthchaft arbeiteten, zur gleichen Zeit Scharlachkranke verpflegten.

Sanitary Record (10. Aug. 1875) berichtet über eine Scharlachepidemie in New Barnet. In den wenigen Tagen vom 29. April bis 7. Mai erkrankten nicht weniger als 128 Personen, im Ganzen wurden 140 Erkrankungsfälle beobachtet, 58 Fälle von Scarlatina kamen in 35 Häusern vor, welche sämmtlich ihre Milch von einem Manne her geliefert erhielten. Einen ähnlichen Bericht bringt dasselbe Journal im Jahre 1879 (15. Juli). Dr Jacob machte in Dorking folgende Beobachtung: Am 26. Mai 1878 erkrankte das Kind eines Ackerarbeiters, dessen andere Kinder in einem anderen Theile des Landes Scharlach durchgemacht hatten und dann nach Hause gekommen waren, ebenfalls an Scharlach. Am 29. erkrankte ein Kind des Nachbars, welches mit dem erkrankten Kinde in Berührung gekommen wal, an Scharlach. Zwischen 1. u..7. Juni ereigneten sich weitere 15 Fälle in drei anderen entfernter wohnenden Familien, welche mit den erkrankten in keiner Verbindung standen, doch bezogen sie sämmtlich die Milch von einem Verschleisser, in dessen Dienst der Vater des oben erwähnten Kindes stand. Dieser letztere, welcher selbst nicht erkrankt war, hatte die Kühe zu melken und that dies auch während der Krankheit des Kindes. Nach Dr. Jacob's Ansicht erscheint es wahrscheinlich, dass das specifische Scharlachgift bei dieser Procedur in die Milch gelangt war und zu den weiteren Erkrankungen Anlass gegeben hatten. Mehrere Kunden, welch abgerahmte Milch aus derselben Quelle bezogen hatten, entgingen der Er krankung. Also auch hier scheint in dem Milchrahm vorzugsweise die Ansammlung des Infectionskeimes stattgefunden zu haben.

In seinem Buche „Die epidemische Verbreitung des Scharlachfiebers in Norwegen berichtet Johannesen kurz: Im Jahre 1874 wird Scharlachfieber durch Milch nach Molde gebracht.

Auch Vacher in Birkenhead (Sanitary Record 1882) betont in einer Mittheilung bezüglich der Uebertragung von Krankheiten durch Kuhmilch, dass Scharlach sicherlich durch die Milchkanne übertragen werden könne.

\section{(Fortsetzung folgt.)}

2. Dem Bundesrath ist die von einer Specialcommission entworfene Anweisung zur Gewinnung, Aufbewahrung und Versendung von Thierlymphe zugegangen. Die Commission empfiehlt ferner, dass über die Thätigkeit der Anstalten zur Gewinnung der Lymphe regelmässige Jahresberichte erstattet und nach einheitlicher Bearbeitung im Gesundheitsamt veröffentlicht werden, sowie dass eine schärfere Ueberwachung des in Apotheken betriebenen Handels mit Lymphe stattfinde.

Nachträglich ist im Reichstage noch ein vom Abgeordneten Dr. Haarmann erstatteter umfangreicher Bericht der Petitionscommission über die Impffrage ausgegeben worden. Wie bekannt, lautet der Antrag der Commission auf Uebergang zur Tagesordnung über die Petitionen gegen den Impfzwang.

3. Flussbäder. Das Corresp.-Bl. für Schweizer Aerzte macht auf folgenden beherzigenswerthen Paragraphen des neuen schweizerischen Lehrbuches für die Sanitätsmannschaft aufmerksam:

Wenn ein Mann beim kalten Baden plötzlich eine auffällige Röthung der Körperhaut zeigt, so ist dies auch bei völligem Wohlbefinden ein sicherer Vorbote einer Ohnmacht, welche leicht zum Ertrinken führt. Der Sanitätssoldat hat solche Leute sofort zu veranlassen, aus dem Wasser zu steigen und sich anzukleiden und bei Weigerung dem militärischen. Vorgesetzten Meldung zu machen."

Diese Vorschrift wurde auf vielfache Erfahrungen namentlich französischer Militärärzte in's Lehrbuch aufgenommen. Dieser Tage ist ein solcher Fall bei einem Soldaten vorgekommen, der im Wasser "krebsroth“, aber wohl war und am Ufer nachher einen schweren Collaps bekam, der auch im seichten Wasser zum Ertrinken geführt bätte. Mancher schwer erklärliche Todesfall bei guten Schwimmern wird verhütet werden können, wenn diesem Vorboten einer Ohnmacht seitens der Mitbadenden die nöthige Aufmerksamkeit geschenkt und der Betreffende, der an nichts Schlimmes denkt, an's Land zu gehen energisch veranlasst wird.

\section{Epidemiologie}

Cholera. Italien. Der Hauptheerd der Seuche ist immer noch die Umgebung von Brilldisi, während in der Provinz Venetien nur vereinzelte Fälle auftreten. Die neusten Depeschen melden folgende Erkrankungen (Todesfälle):

$\begin{array}{rcccccc}\text { Juli } & \text { Brindisi } & \text { Latiano } & \text { Francavilla } & \text { San Vito } & \text { Oria } & \text { Erchie } \\ 6 .-7 . & 8(5) & 70(26) & 76(34) & 32(4) & 3(1) & 12(2) \\ 7 .-8 . & 7(4) & 45(21) & 49(19) & 27(4) & - & 8(1) \\ 8 .-9 . & 12(7) & 52(22) & 47(41) & - & 4(1) & 12(3) \\ 9 .-10 . & 15(5) & 32(15) & 59(21) & 12(3) & 1(1) & 14 \\ 10-11 . & 8(3) & 28(8) & 52(33) & 15(3) & - & - \\ 11 .-12 . & 1(2) & 6(10) & 76(16) & 0(2) & - & 11(4) \\ 12 .-13 . & - & 33(12) & 73(26) & 17(4) & 0(1) & 20(5)\end{array}$
Ostuni.

Ausserdem sind einzelne Erkrankungen vorgekommen in Messagne und

Oesterreich. Nachdem schon in den früheren Tagen vereinzelte Fälle aus Triest und Fiume gemeldet wurden, bringt das Reuter'sche Bureau vom 12. Juli die Nachricht, dass vom Mittag des 11. bis Mittag des 12. Juli in Triest 8 Erkrankungen und 2 Todesfälle vorgekommen sind, darunter 2 (1) Militärpersonen. In Agram hat eine Conferenz im Schoosse der Landesregierung stattgefunden, in der beschlossen wurde, einen Cordon um Fiume, Sasate und Trsato zu ziehen und überall an der Linie Desinfectionsanstalten zu errichten. 